\title{
Health Literacy and Quality of Care of Patients with Diabetes: A Cross-Sectional Analysis
}

Emilie Zuercher $^{a^{*}}$, Ibrahima D. Diatta ${ }^{a}$, Bernard Burnand ${ }^{a}$, Isabelle Peytremann-Bridevaux ${ }^{a}$

\section{Abstract}

Background: Limited health literacy $(\mathrm{HL})$ may lead to poor health outcomes and inappropriate healthcare use, particularly in patients with chronic diseases. We aimed to assess the association between functional $\mathrm{HL}(\mathrm{FHL})$ and quality of care, as measured by process- and outcome-of-care indicators, in patients with diabetes. 
Methods: This cross-sectional study used data from the 2013 CoDiab-VD cohort follow-up, which included non-institutionalised adults with diabetes from canton of Vaud, Switzerland. Using self-administered questionnaires, we collected patients' characteristics, processes [annual HbA1C check, lipid profile, urine test, foot examination, influenza vaccination, eye examination (24 months), physical activity and diet recommendations] and outcomes of care (HbA1C knowledge, HbA1C value, SF-12, ADDQoL, PACIC, self-efficacy). A single validated screening question assessed $\mathrm{FHL}$. Unadjusted and adjusted regression analyses were performed.

Results: Of 381 patients $52.5 \%$ (95\% Cl: $47.5 \%-57.5 \%), 40.7 \%(95 \% \mathrm{Cl}: 35.7 \%-45.6 \%)$ and $6.8 \%(95 \% \mathrm{Cl}: 4.3 \%-9.4 \%)$ reported high, medium and poor FHL, respectively. Significant associations were found for two out of seven outcomes of care; lower self-efficacy scores associated with medium and poor $\mathrm{FHL}$ (adjusted: $\beta-0.6,95 \% \mathrm{Cl}-0.9$ to -0.2 and $\beta-1.8,95 \% \mathrm{Cl}-$ 2.5 to -1.2 , respectively), lower SF-12 mental scores associated with poor FHL (adjusted: $\beta-8.4$, $95 \% \mathrm{Cl}-12.5$ to -4.2$)$.

Conclusions: This study found few outcomes of care associated with FHL. Further exploration of the impact of limited $\mathrm{HL}$ on quality of care indicators will help tailor initiatives - both on patients' and providers' side - to improve diabetes care.

\section{Keywords}

Health literacy, Diabetes, Quality of care 


\section{Introduction}

The burden of diabetes continues to increase worldwide, with more than 550 million people predicted to be affected by this disease in 2030 [1]. To manage their chronic condition daily, people with diabetes need self-care and self-efficacy skills. With the increasing focus on patientcentred care and patient empowerment of patients with chronic conditions, health literacy $(\mathrm{HL})$ emerges as an important aspect to consider. Indeed, lower HL has not only been shown to be a barrier to optimum self-management and efficient empowerment [2], but has also be shown to be associated with suboptimal health and care outcomes [3]. Health literacy can be defined as "the degree to which individuals can obtain, process, understand, and communicate about health-related information needed to make informed health decisions" [4]. It is a multidimensional concept, whose three dimensions range from one that requires more basic skills to one that requires more complex skills: functional, interactive and critical HL. Functional HL (FHL), which focuses on "the basic skills in reading and writing that are necessary to function effectively in everyday situations" [5, 6], has been the most investigated dimension. Many validated instruments measuring this dimension exist, including brief instruments that allow for easy use in self-administered questionnaires [7]. Until now, published studies targeting patients with diabetes, which have focused mainly on the association between HL and patients' health behaviours (e.g. self-care), diabetes knowledge or diabetes clinical intermediate outcomes, such as $\mathrm{HbA} 1 \mathrm{C}$ and LDL-cholesterol levels, have shown mixed results $[3,8,9]$. The effect of $H L$ on processes of care and other patient-reported outcomes has more rarely been investigated. In that context, the aim of our study was, first, to measure the level of one dimension of $\mathrm{HL}$ (i.e. FHL) among patients with diabetes and, second, to explore the association between FHL and outcomes less often considered until now: selfreported process and outcome of care indicators.

\section{Methods}

\section{Study design and population}

This cross-sectional study was based on data from the 2013 follow-up questionnaire of the CoDiab-VD cohort [10].This prospective cohort study, launched in 2011 in the canton of Vaud, Switzerland, recruited patients with diabetes visiting community-based pharmacies with a diabetes-related prescription during two six-week periods in 2011 and 2012. 
Eligible patients were non-institutionalised adults ( $\geq 18$ years) with diabetes of at least one year duration, residing in the canton of Vaud, with a sufficient level of French to complete a questionnaire and without cognitive impairment or gestational diabetes. Participants were then followed up annually, filling in a self-reported paper questionnaire investigating various aspects of living with diabetes [10, 11]. Among the 519 patients recruited in 2011-2012, we sent the 2013 follow-up questionnaire to the 449 patients who were not lost to follow-up. Among the 395 patients who sent the questionnaire back, 381 answered the FHL question and were considered in the analyses.

\section{Data}

\section{Main exposure (independent) variable: Health literacy}

Health literacy $(\mathrm{HL})$ was measured by using the validated French version [12] of a single screening question assessing FHL [7], which had been shown to have good sensitivity and specificity to detect people with $\mathrm{HL}$ limitations [7, 13]. Participants responded to the 5-point Likert scale question: "When you get written information on a medical treatment or your medical condition, how often do you have problems understanding what it is telling you?" [12]. Responses were divided into three categories: a good level of FHL (never having problems), a medium level of FHL (occasionally or sometimes having problems) and a poor level of $\mathrm{FHL}$ (often or always having problems).

\section{Dependent variables: Quality of care indicators}

The quality of care indicators targeted were both processes and outcomes of care. The processes of care for the past 12 months that we considered were: the number of times that the HbA1C level was checked among HbA1C-aware patients (1x, $\geq 2 x$, none, do not know), the number of times a lipid profile was done (1x, 2-3x, $\geq 4 x$, none, do not know), whether a urine test for microalbuminuria was done (yes, no, do not know), whether a foot examination was performed by a healthcare professional (yes, no, do not know), whether an eye examination was performed by an ophthalmologist (<1 year ago, 1-2 years ago, >2 years ago, never, do not know) and whether the patient received an influenza vaccination (yes, no, do not know). We also considered the following two processes of care without a time frame: whether patients had received any physical activity recommendations (yes, no, do not know) and written or verbal diet recommendations (yes, no, do not know All processes of care variables were dichotomised as patients having received at least one check versus no check. The "do not know" answers were 
considered as missing values. For example, if patients answered " $1 \mathrm{x}$ ", "2-3x" or " $\geq 4 \mathrm{x}$ " for lipid profile, they were categorised as having received one check; those answering "none" were categorised as having received no check; and those answering "do not know" were categorised as missing values and excluded from the denominator. The outcomes of care considered were: $\mathrm{HbA1C}$ awareness (yes, no, do not know), the patient's reported HbA1C level, (health-related) quality of life as measured by a generic questionnaire [12-item Short Form Health Survey (SF12): physical component score (PCS) and mental component score (MCS), range: $0=$ worst score to 100 = best score; scores constructed to have a mean of 50 and a standard deviation of 10 in the general US population] [14] and a diabetes-specific questionnaire [Audit of DiabetesDependent Quality of Life 19 (ADDQoL), range: -9 = maximum negative impact of diabetes to +3 = maximum positive impact of diabetes] [15], the patient's assessment of how care is congruent with the Chronic Care Model recommendations (Patient Assessment of Chronic Illness Care $(\mathrm{PACIC})$ questionnaire; range: $1=$ lowest score to $5=$ highest score) $[16,17]$, and self-efficacy using the Stanford self-efficacy questionnaire (range: $1=$ lowest confidence to $10=$ highest confidence) [18].

\section{Other variables}

The other variables included in this study were gender, age, education, household income in $\mathrm{CHF} /$ month, type of diabetes, diabetes treatment, duration of diabetes, smoking status, physical activity levels using questions from the Swiss health Survey [19], at-risk alcohol consumption using the Alcohol Use Disorders and Identification Test-Consumption questionnaire (men $\geq 4$, women $\geq 3$ ) [20], body mass index (weight in kilograms divided by the squared height in meters), number of diabetes-related complications and number of comorbidities.

\section{Statistical analyses}

We conducted bivariate analyses, using Fisher's exact test for categorical variables and t-test for continuous variables, to characterise the population under study according to FHL levels. A nonparametric test for trend across ordered groups was then used to assess the linear relationship between process- and outcome-of-care and levels of FHL (nptrend command in Stata). We performed unadjusted and adjusted regression analyses (logistic and linear for dichotomous and continuous variables, respectively) to assess whether FHL predicted quality of care indicators (processes and outcomes of care). The main potential confounders selected a priori in the adjusted analyses, based on the literature, were: age, gender, education, diabetes 
treatment, type of diabetes and number of comorbidities. Because of the limited number of participants and events, we restricted the number of coefficients in regression models to nine, following the rule of thumb of van Belle, which suggests that at least 10 events per investigated variable are necessary in logistic regressions [21]. We assessed the full models for influential observations, and removed them from the final models. For logistic regressions, the calibration of final models was tested using Hosmer-Lemeshow goodness-of-fit test. For linear regressions, the validity of final models was checked with the root mean square error (root MSE).

Due to the too small number of events in the category "not done" as well as the fact that HbA1C analyses were restricted to patients aware of what $\mathrm{HbA} 1 \mathrm{C}$ was, the $\mathrm{HbA} 1 \mathrm{C}$ check variable was not considered as an outcome in the regression analyses.

All analyses were performed using Stata 13.1 (Stata Corporation, College Station, TX, USA, http://www.stata.com).

\section{Ethical considerations}

The study protocol was reviewed and approved by the Cantonal Ethics Committee of Research on Human Beings of the canton of Vaud (Protocol $N^{\circ} 151 / 11$ ), and the CoDiab-VD cohort was registered with ClinicalTrials.gov, identifier NCT01902043. Written informed consent was obtained from all participants, and data are being kept confidential and anonymous.

\section{Results}

Characteristics of the 381 participants having answered the FHL question are presented in Table 1 . They were mainly male (60\%), their mean age was 65 years and $88 \%$ had type 2 diabetes.

Comparing baseline characteristics of the 381 respondents with $\mathrm{FHL}$ data to those of the 138 patients without FHL data, patients' characteristics were not different in terms of gender, education, household income or type of diabetes, but patients with FHL data were slightly younger at recruitment than patients without $\mathrm{FHL}$ data (63.7 vs 66.6 years, $p=0.008$; data not shown).

Half of the participants $(52.5 \%, 95 \% \mathrm{Cl}: 47.5 \%-57.5 \%)$ reported never having problems understanding medical information (good $\mathrm{FHL})$, whereas $40.7 \%(95 \% \mathrm{Cl}: 35.7 \%-45.6 \%)$ reported having problems occasionally or sometimes (medium $\mathrm{FHL}$ ) and $6.8 \%$ (95\% Cl: $4.3 \%-9.4 \%)$ often 
or always (poor FHL). Differences among FHL groups were observed for household incomes ( $p$ 0.043 ) and duration of diabetes ( $p$ 0.032); patients with low level of FHL reporting more often lower incomes and longer duration of diabetes.

Among the eight process-of-care indicators investigated, no significant differences were observed across the three groups of $\mathrm{FHL}$, except for physical activity recommendation (increasing report of recommendations across decreasing FHL levels; Table 2). For four out of seven outcome-of-care indicators - SF-12 MCS, SF-12 PCS, ADDQoL global score and selfefficacy score - a significant trend was observed with scores increasing with increasing $\mathrm{FHL}$ levels.

Results of crude and adjusted regression analyses suggested that the receipt of processes of care -lipid profile, urine test, foot examination, eye examination and influenza vaccination individually, as well as diet recommendations - were not associated with FHL (Table 3). These analyses nevertheless suggested that patients with medium FHL more often received physical activity recommendations than did patients with good FHL when adjusted for potential confounders (adjusted OR 1.9, 95\% Cl 1.1 to 3.1). Hosmer and Lemeshow's goodness-of-fit tests showed good fit of the data.

Whereas unadjusted analyses of four out of seven outcomes of care presented significantly lower scores for patients with poor FHL than for those with good FHL (SF-12 MCS: $\beta-9.1$, $95 \% \mathrm{Cl}-13.5$ to -4.8 ; SF-12 PCS: $\beta-4.9,95 \% \mathrm{Cl}-8.9$ to -0.8 ; ADDQoL global score: $\beta-1.1$, $95 \% \mathrm{Cl}-1.7$ to -0.5 ; self-efficacy score: $\beta-1.7,95 \% \mathrm{Cl}-2.4$ to -1.0 ) (Table 4 ), this trend persisted only for the SF-12 MCS $(\beta-8.4,95 \% \mathrm{Cl}-12.5$ to -4.2$)$ and the self-efficacy score $(\beta-1.8,95 \% \mathrm{Cl}$ 2.5 to -1.2$)$ after adjustment for potential confounding factors. The self-efficacy score was also significantly lower in patients with medium FHL than for patients with good FHL in unadjusted ( $\beta$ $-0.6,95 \% \mathrm{Cl}-0.9$ to -0.2$)$ and adjusted models $(\beta-0.6,95 \% \mathrm{Cl}-0.9$ to -0.2$)$. After analysing the regression residuals and checking for influential observations, the latter were removed from the models; the final models showed good validity.

Similar results were obtained when we dichotomised the FHL variable into "no problem to understand medical information" (corresponding to the good FHL category) and "any problem" (corresponding to low and medium FHL categories together). Regression analyses suggest that physical activity recommendations were more often given to patients reporting "any problem" 
compared to patients without FHL problems (adjusted OR 1.9, 95\% Cl 1.2 to 3.1). They also suggest lower SF-12 MCS scores (adjusted $\beta-2.5,95 \% \mathrm{Cl}-4.6$ to -0.4 ) and self-efficacy scores (adjusted $\beta-0.7,95 \% \mathrm{Cl}-1.0$ to -0.4 ) in patients with $\mathrm{FHL}$ problems compared to patients without problems.

\section{Discussion}

In this study, we measured the level of FHL among patients with diabetes and assessed the relationship between FHL and quality-of-care indicators. First, our results showed that almost half of the participants of the CoDiab-VD cohort reported having good FHL and that less than a tenth self-reported poor FHL. We then observed that although the receipt of processes of care in the past 12 months was not associated with $\mathrm{FHL}$-except for physical activity recommendations-, a few significant associations between FHL and outcomes of care were observed, with lower self-efficacy and SF-12 MCS scores reported by patients with poorer FHL. The prevalence of good FHL of approximately $50 \%$ is consistent with those of another study involving people with diabetes residing in the three main Swiss linguistic regions and that used the same FHL instrument [12]. It is also close to those from several US studies that used the same screening question, in which prevalence of good $\mathrm{FHL}$ varied between $39 \%$ and $53 \%$, despite the fact that various population and healthcare context were considered [22-24].

The absence of an association between most process-of-care indicators and FHL levels is in line with the results of Mounce et al. [25] that showed no differences between patients with low and high $\mathrm{HL}$ regarding the receipt of a care bundle comprising annual $\mathrm{HbA} 1 \mathrm{C}$ and proteinuria checks, as well as annual foot examination. However, whereas we found no differences among patients with different FHL levels for the annual influenza vaccination, other studies that mostly targeted elderly people showed that people with lower HL levels were less likely to report having received an influenza vaccination during the previous 12 months [26, 27] or ever having had influenza vaccination $[3,28]$. In addition, whereas we did not find a significant relationship between FHL and long-term glycaemic control in our study, mixed results were reported by other studies, with some finding higher $\mathrm{HbA} 1 \mathrm{C}$ values among patients with low $\mathrm{HL}$ and others showing no association [8, 9]. These non-consistent results of the association between FHL and processes of care could be explained by the fact that the latter may be less dependent on the patients' level of FHL than on the actions of healthcare practitioners [29]. Moreover, the influence of patients on such actions involves communication skills that might be better captured 
by interactive measures than by $\mathrm{FHL}$ measures $[5,6]$, interactive $\mathrm{HL}$ not having been investigated in our study.

The absence of an association between $\mathrm{FHL}$ and processes of care contrasts with the significant FHL and outcome-of-care associations: higher FHL levels were associated with better selfefficacy scores and with better health-related quality-of-life mental summary scores. Whether these significant results represent true associations remains uncertain, however. Indeed, whereas higher levels of self-efficacy or better $\mathrm{HbA} 1 \mathrm{C}$ control may be expected in patients reporting high $\mathrm{FHL}[2,30]$, published results of the latter association and of the associations between FHL and health-related quality of life, vary considerably. In fact, although a positive effect of FHL on diabetes self-efficacy was found in some studies [2, 30-32], no association was found between self-efficacy and HL in other studies [33-35]. In addition, whereas some researchers reported a positive association between $\mathrm{FHL}$ and health-related quality-of-life mental scores [36, 37], other investigators did not observe any relationship between these two dimensions [38, 39]. These discrepancies, which call for caution when interpreting the results, may be due to: differences in the methods used to measure HL levels and the outcomes; diversity of populations included; differences in source populations; and use of a single-item instrument which did not allow a comprehensive measure of $\mathrm{HL}$, a complex concept that also includes interactive and critical dimensions $[5,6]$, even though the single HL screening question had been shown to be a valid measure of $\operatorname{FHL}[7,13]$.

Whereas the main strength of our study was the fact that we explored the association between $\mathrm{FHL}$ and a broad range of quality-of-care indicators, these results need to be interpreted considering the following limitations. First, only persons fluent enough in French to fill in a paper questionnaire were included in the CoDiab-VD cohort, leaving those who did not speak French, who were illiterate or who had limited HL, underrepresented in our sample. How the inclusion of the latter patients would have modified the size and direction of the associations remains unknown, even if we may have a priori hypotheses suggesting point estimate increases. Despite of that, CoDiab-VD participants were relatively similar, at baseline, in terms of age, gender, education, smoking status and BMI, to those of participants with diabetes of the CoLaus study, a population-based study conducted in the same area and period [40]. Second, our sample included a small number of patients reporting low FHL, possibly underpowering our analyses. In addition, we cannot exclude that the significant associations occurred by chance due to the number of statistical analyses performed. Third, the study design was cross-sectional, which precludes us from inferring any causative association between FHL and processes or outcomes of care. We also used patients' self-reported data, which may be prone to recall bias or may 
cause low health literate patients to underreport medical information; specific analyses, however, demonstrated good agreement between patient- and physician-reported outcomes [41]. In addition, although future longitudinal analyses may help us to understand whether FHL is associated with more distal health outcomes, we used self-reported patient-centred outcomes, which are increasingly being considered as key in the evaluation of patients' care. Fourth, generalising our results to other patients with diabetes, especially those residing in other parts of Switzerland, should be done with caution since differences in the prevalence of adequate FHL among the general population were found across the three linguistic regions of the country $(67 \%$ in the Italian-speaking area, 83\% in the French-speaking area and 93\% in the German-speaking area) [42], and we included only those people residing in a French-speaking canton of Switzerland. Our prevalence results were, however, close to those of Franzen et al. [12], who also investigated $\mathrm{FHL}$ in people with diabetes and across the three linguistic regions of Switzerland.

\section{Conclusion}

The results of this study suggested an association between $\mathrm{HL}$ levels and few outcomes of care. Contrary to the numerous studies on the relationship between $\mathrm{HL}$ and outcomes of care, the influence of $\mathrm{HL}$ on the receipt of processes of care has been poorly investigated. Further exploration of the impact of limited HL, taking into account its three dimensions, on process and outcomes of care, will help tailor initiatives - both on patients' and providers' side - to improve diabetes care.

\section{Acknowledgements}

We wish to thank the participants of the CoDiab-VD cohort who participated in the study, especially those who participated in the 2013 follow-up. We also thank the partners and members of the working groups for their collaboration, as well as Ms. Tania Carron for a critical reading of the manuscript.

This research was presented orally at the Swiss Public Health Conference 2014 in Olten, This project was funded by the Department of Public Health of the canton of Vaud ("Programme cantonal Diabète"). Prof. I. Peytremann-Bridevaux was supported by a grant from the Swiss National Science Foundation [PROSPER N³2333B-123817 and N³2333B-139789] to July 2013 and is currently supported by the Swiss School of Public Health+ [Assistant Professorship 
grant]. The funders had no role in study design, data collection, analysis and interpretation, decision to publish results, and/or preparation of the manuscript.

\section{References}

[1] D.R. Whiting, L. Guariguata, C. Weil, J. Shaw, IDF diabetes atlas: global estimates of the prevalence of diabetes for 2011 and 2030, Diabetes Res Clin Pract, 94 (2011) 311-321.

[2] M.P. Fransen, C. von Wagner, M.L. Essink-Bot, Diabetes self-management in patients with low health literacy: ordering findings from literature in a health literacy framework, Patient education and counseling, 88 (2012) 44-53.

[3] N.D. Berkman, S.L. Sheridan, K.E. Donahue, D.J. Halpern, K. Crotty, Low health literacy and health outcomes: an updated systematic review, Ann Intern Med, 155 (2011) 97-107.

[4] N.D. Berkman, T.C. Davis, L. McCormack, Health literacy: what is it?, Journal of health communication, 15 Suppl 2 (2010) 9-19.

[5] D. Nutbeam, The evolving concept of health literacy, Soc Sci Med, 67 (2008) 2072-2078.

[6] K. Sorensen, S. Van den Broucke, J. Fullam, G. Doyle, J. Pelikan, Z. Slonska, H. Brand, E.

Consortium Health Literacy Project, Health literacy and public health: a systematic review and integration of definitions and models, BMC Public Health, 12 (2012) 80.

[7] L.D. Chew, J.M. Griffin, M.R. Partin, S. Noorbaloochi, J.P. Grill, A. Snyder, K.A. Bradley, S.M. Nugent, A.D. Baines, M. Vanryn, Validation of screening questions for limited health literacy in a large VA outpatient population, Journal of general internal medicine, 23 (2008) 561-566.

[8] F. Al Sayah, S.R. Majumdar, B. Williams, S. Robertson, J.A. Johnson, Health literacy and health outcomes in diabetes: a systematic review, Journal of general internal medicine, 28 (2013) 444-452.

[9] S.C. Bailey, A.G. Brega, T.M. Crutchfield, T. Elasy, H. Herr, K. Kaphingst, A.J. Karter, S. Moreland-Russell, C.Y. Osborn, M. Pignone, R. Rothman, D. Schillinger, Update on health literacy and diabetes, The Diabetes educator, 40 (2014) 581-604.

[10] E. Zuercher, J. Bordet, B. Burnand, I. Peytremann-Bridevaux, CoDiab-VD: protocol of a prospective population-based cohort study on diabetes care in Switzerland, BMC Health Serv Res, 15 (2015) 329.

[11] I. Peytremann-Bridevaux, J. Bordet, V. Santschi, T.H. Collet, M. Eggli, B. Burnand, Community-based pharmacies: an opportunity to recruit patients?, International journal of public health, (2013).

[12] J. Franzen, S. Mantwill, R. Rapold, P.J. Schulz, The relationship between functional health literacy and the use of the health system by diabetics in Switzerland, European journal of public health, (2013).

[13] F. Al Sayah, S.R. Majumdar, L.E. Egede, J.A. Johnson, Measurement properties and comparative performance of health literacy screening questions in a predominantly low income African American population with diabetes, Patient education and counseling, 97 (2014) 88-95. [14] J.A. Fleishman, A.J. Selim, L.E. Kazis, Deriving SF-12v2 physical and mental health summary scores: a comparison of different scoring algorithms, Quality of life research : an international journal of quality of life aspects of treatment, care and rehabilitation, 19 (2010) 231241.

[15] C. Bradley, C. Todd, T. Gorton, E. Symonds, A. Martin, R. Plowright, The development of an individualized questionnaire measure of perceived impact of diabetes on quality of life: the ADDQoL, Quality of life research : an international journal of quality of life aspects of treatment, care and rehabilitation, 8 (1999) 79-91. 
[16] R.E. Glasgow, H. Whitesides, C.C. Nelson, D.K. King, Use of the Patient Assessment of Chronic Illness Care (PACIC) with diabetic patients: relationship to patient characteristics, receipt of care, and self-management, Diabetes Care, 28 (2005) 2655-2661.

[17] K. Iglesias, B. Burnand, I. Peytremann-Bridevaux, PACIC Instrument: disentangling dimensions using published validation models, International journal for quality in health care : journal of the International Society for Quality in Health Care / ISQua, 26 (2014) 250-260. [18] K. Lorig, P.L. Ritter, F.J. Villa, J. Armas, Community-based peer-led diabetes selfmanagement: a randomized trial, The Diabetes educator, 35 (2009) 641-651.

[19] Swiss Health Survey, in.

[20] K. Bush, D.R. Kivlahan, M.B. McDonell, S.D. Fihn, K.A. Bradley, The AUDIT alcohol consumption questions (AUDIT-C): an effective brief screening test for problem drinking. Ambulatory Care Quality Improvement Project (ACQUIP). Alcohol Use Disorders Identification Test, Archives of internal medicine, 158 (1998) 1789-1795.

[21] G. van Belle, Statistical rules of thumb, Wiley-Interscience, New York, 2002.

[22] U. Sarkar, A.J. Karter, J.Y. Liu, N.E. Adler, R. Nguyen, A. Lopez, D. Schillinger, The literacy divide: health literacy and the use of an internet-based patient portal in an integrated health system-results from the diabetes study of northern California (DISTANCE), Journal of health communication, 15 Suppl 2 (2010) 183-196.

[23] U. Sarkar, A.J. Karter, J.Y. Liu, H.H. Moffet, N.E. Adler, D. Schillinger, Hypoglycemia is more common among type 2 diabetes patients with limited health literacy: the Diabetes Study of Northern California (DISTANCE), Journal of general internal medicine, 25 (2010) 962-968.

[24] U. Sarkar, J.D. Piette, R. Gonzales, D. Lessler, L.D. Chew, B. Reilly, J. Johnson, M. Brunt, J. Huang, M. Regenstein, D. Schillinger, Preferences for self-management support: findings from a survey of diabetes patients in safety-net health systems, Patient education and counseling, 70 (2008) 102-110.

[25] L.T. Mounce, N. Steel, A.C. Hardcastle, W.E. Henley, M.O. Bachmann, J.L. Campbell, A. Clark, D. Melzer, S.H. Richards, Patient characteristics predicting failure to receive indicated care for type 2 diabetes, Diabetes Res Clin Pract, 107 (2015) 247-258.

[26] R.L. Sudore, K.M. Mehta, E.M. Simonsick, T.B. Harris, A.B. Newman, S. Satterfield, C. Rosano, R.N. Rooks, S.M. Rubin, H.N. Ayonayon, K. Yaffe, Limited literacy in older people and disparities in health and healthcare access, Journal of the American Geriatrics Society, 54 (2006) 770-776.

[27] I.M. Bennett, J. Chen, J.S. Soroui, S. White, The contribution of health literacy to disparities in self-rated health status and preventive health behaviors in older adults, Annals of family medicine, 7 (2009) 204-211.

[28] T.L. Scott, J.A. Gazmararian, M.V. Williams, D.W. Baker, Health literacy and preventive health care use among Medicare enrollees in a managed care organization, Medical care, 40 (2002) 395-404.

[29] N.S. Morris, C.D. MacLean, B. Littenberg, Literacy and health outcomes: a cross-sectional study in 1002 adults with diabetes, BMC family practice, 7 (2006) 49.

[30] L.D. Woodard, C.R. Landrum, A.B. Amspoker, D. Ramsey, A.D. Naik, Interaction between functional health literacy, patient activation, and glycemic control, Patient preference and adherence, 8 (2014) 1019-1024.

[31] C.Y. Osborn, K. Cavanaugh, K.A. Wallston, R.L. Rothman, Self-efficacy links health literacy and numeracy to glycemic control, Journal of health communication, 15 Suppl 2 (2010) 146-158. [32] W. Bohanny, S.F. Wu, C.Y. Liu, S.H. Yeh, S.L. Tsay, T.J. Wang, Health literacy, selfefficacy, and self-care behaviors in patients with type 2 diabetes mellitus, Journal of the American Association of Nurse Practitioners, 25 (2013) 495-502.

[33] U. Sarkar, L. Fisher, D. Schillinger, Is self-efficacy associated with diabetes selfmanagement across race/ethnicity and health literacy?, Diabetes Care, 29 (2006) 823-829. 
[34] D.A. DeWalt, R.S. Boone, M.P. Pignone, Literacy and its relationship with self-efficacy, trust, and participation in medical decision making, American journal of health behavior, 31 Suppl 1 (2007) S27-35.

[35] V. McCleary-Jones, Health literacy and its association with diabetes knowledge, selfefficacy and disease self-management among African Americans with diabetes mellitus, The ABNF journal : official journal of the Association of Black Nursing Faculty in Higher Education, Inc, 22 (2011) 25-32.

[36] I. van der Heide, J. Wang, M. Droomers, P. Spreeuwenberg, J. Rademakers, E. Uiters, The relationship between health, education, and health literacy: results from the Dutch Adult Literacy and Life Skills Survey, Journal of health communication, 18 Suppl 1 (2013) 172-184.

[37] F. Al Sayah, W. Qiu, J.A. Johnson, Health literacy and health-related quality of life in adults with type 2 diabetes: a longitudinal study, Quality of life research : an international journal of quality of life aspects of treatment, care and rehabilitation, (2015).

[38] F. Al Sayah, S.R. Majumdar, J.A. Johnson, Association of Inadequate Health Literacy with Health Outcomes in Patients with Type 2 Diabetes and Depression: Secondary Analysis of a Controlled Trial, Can J Diabetes, (2015).

[39] D.H. Howard, T. Sentell, J.A. Gazmararian, Impact of health literacy on socioeconomic and racial differences in health in an elderly population, Journal of general internal medicine, 21 (2006) 857-861.

[40] M. Firmann, V. Mayor, P.M. Vidal, M. Bochud, A. Pecoud, D. Hayoz, F. Paccaud, M. Preisig, K.S. Song, X. Yuan, T.M. Danoff, H.A. Stirnadel, D. Waterworth, V. Mooser, G. Waeber, P. Vollenweider, The CoLaus study: a population-based study to investigate the epidemiology and genetic determinants of cardiovascular risk factors and metabolic syndrome, BMC cardiovascular disorders, 8 (2008) 6.

[41] T.H. Collet, P. Taffe, J. Bordet, B. Burnand, I. Peytremann-Bridevaux, Reproducibility of diabetes quality of care indicators as reported by patients and physicians, European journal of public health, 24 (2014) 1004-1009.

[42] M. Connor, S. Mantwill, P.J. Schulz, Functional health literacy in Switzerland--validation of a German, Italian, and French health literacy test, Patient education and counseling, 90 (2013) 1217. 
Table 1: Participants' overall characteristics and by functional health literacy level

\begin{tabular}{|c|c|c|c|c|c|}
\hline & $\begin{array}{l}\text { Total } \\
(n=381)\end{array}$ & $\begin{array}{l}\text { Good } \\
(n=200)\end{array}$ & $\begin{array}{l}\text { Medium } \\
(n=155)\end{array}$ & Poor $(n=26)$ & $p$-value \\
\hline Male $(n=381)$ & $60.6 \%$ & $62.0 \%$ & $61.3 \%$ & $46.2 \%$ & 0.298 \\
\hline Age (year) (n=381) & $65.2 \pm 10.8$ & $64.8 \pm 11.3$ & $66.0 \pm 10.0$ & $63.8 \pm 11.2$ & 0.453 \\
\hline Education $(n=372)$ & & & & & 0.082 \\
\hline Compulsory school or less (I) & $17.2 \%$ & $12.8 \%$ & $20.7 \%$ & $30.8 \%$ & \\
\hline $\begin{array}{l}\text { Vocational training or high } \\
\text { school (II) }\end{array}$ & $56.2 \%$ & $57.7 \%$ & $54.7 \%$ & $53.9 \%$ & \\
\hline $\begin{array}{l}\text { University or technical college } \\
\text { (III) }\end{array}$ & $26.6 \%$ & $29.6 \%$ & $24.7 \%$ & $15.4 \%$ & \\
\hline $\begin{array}{l}\text { Household income } \\
\text { (CHF/month) (n=338) }\end{array}$ & & & & & 0.043 \\
\hline$<3599$ & $21.0 \%$ & $18.8 \%$ & $23.7 \%$ & $23.8 \%$ & \\
\hline $3600-5899$ & $33.1 \%$ & $28.5 \%$ & $38.2 \%$ & $42.9 \%$ & \\
\hline $5900-9599$ & $28.4 \%$ & $36.0 \%$ & $19.1 \%$ & $19.1 \%$ & \\
\hline$>9600$ & $17.5 \%$ & $16.7 \%$ & $19.1 \%$ & $14.3 \%$ & \\
\hline Type of diabetes $(n=381)$ & & & & & 0.168 \\
\hline Type 1 & $12.3 \%$ & $14.0 \%$ & $9.0 \%$ & $19.2 \%$ & \\
\hline Type $2+$ other $^{\text {a }}$ & $87.7 \%$ & $86.0 \%$ & $91.0 \%$ & $80.8 \%$ & \\
\hline Treatment $(n=375)$ & & & & & 0.811 \\
\hline Oral antidiabetics & $46.4 \%$ & $45.7 \%$ & $48.0 \%$ & $42.3 \%$ & \\
\hline $\begin{array}{l}\text { Insulin or other antidiabetic } \\
\text { injection }\end{array}$ & $53.6 \%$ & $54.3 \%$ & $52.0 \%$ & $57.7 \%$ & \\
\hline Duration of diabetes $(n=376)$ & & & & & 0.032 \\
\hline$\leq 10$ years & $54.8 \%$ & $52.8 \%$ & $60.8 \%$ & $34.6 \%$ & \\
\hline$>10$ years & $45.2 \%$ & $47.2 \%$ & $39.2 \%$ & $65.4 \%$ & \\
\hline Currently smoking $(n=370)$ & $16.5 \%$ & $16.7 \%$ & $16.5 \%$ & $15.4 \%$ & 1.000 \\
\hline Physical inactivity ( $n=372$ ) & $30.1 \%$ & $28.4 \%$ & $31.3 \%$ & $36.0 \%$ & 0.656 \\
\hline $\begin{array}{l}\text { Overweight or obesity (BMI } \\
\left.\geq 25 \mathrm{~kg} / \mathrm{m}^{2}\right)(\mathrm{n}=357)\end{array}$ & $80.7 \%$ & $77.8 \%$ & $82.5 \%$ & $92.0 \%$ & 0.198 \\
\hline $\begin{array}{l}\text { At-risk alcohol consumption }{ }^{\text {b }} \\
(n=366)\end{array}$ & $45.9 \%$ & $45.3 \%$ & $44.7 \%$ & $58.3 \%$ & 0.476 \\
\hline $\begin{array}{l}\text { Number of complications } \\
(n=368)\end{array}$ & $0.7 \pm 0.9$ & $0.7 \pm 0.9$ & $0.7 \pm 1.0$ & $0.6 \pm 0.9$ & 0.874 \\
\hline $\begin{array}{l}\text { Number of comorbidities } \\
(n=375)\end{array}$ & $1.7 \pm 1.3$ & $1.6 \pm 1.3$ & $1.8 \pm 1.3$ & $2.0 \pm 1.3$ & 0.279 \\
\hline
\end{tabular}

Data are presented as the percentage or mean \pm SD. Because of rounding, numbers may not add up to $100 \%$. Pvalues were calculated either with Fisher's exact test for categorical variables or with one-way analysis of variance (ANOVA) for continuous variables.

CHF: Swiss franc; BMI: body mass index.

a: Other type of diabetes were corticosteroid-induced diabetes $(n=2)$, diabetes after pancreatectomy $(n=1)$ and undefined type of diabetes $(n=17)$.

b: Measured with the Alcohol Use Disorders and Identification Test-Consumption (men $\geq 4$, women $\geq 3$ ). 
Table 2: Overall process- and outcome-of-care indicators and by functional health literacy level

\begin{tabular}{|c|c|c|c|c|c|}
\hline & Total $(n=381)$ & Good $(n=200)$ & $\begin{array}{l}\text { Medium } \\
(n=155)\end{array}$ & Poor $(n=26)$ & $\begin{array}{l}\text { P-value } \\
\text { for trend }\end{array}$ \\
\hline Process-of-care indicators & $\%(n)$ & & & & \\
\hline $\begin{array}{l}\text { Annual HbA1C check } \\
\text { done at least } 1 \mathrm{x}\end{array}$ & $98.3 \%(294)$ & $97.5 \%(160)$ & $99.1 \%(116)$ & $100.0 \%(18)$ & 0.242 \\
\hline Annual lipid profile done & $94.3 \%(370)$ & $93.8 \%(193)$ & $94.0 \%(151)$ & $100.0 \%(26)$ & 0.364 \\
\hline Annual urine test done & $79.3 \%(343)$ & $79.1 \%(182)$ & $80.3 \%(137)$ & $75.0 \%(24)$ & 0.880 \\
\hline $\begin{array}{l}\text { Annual foot examination } \\
\text { done }\end{array}$ & $59.0 \%(368)$ & $58.0 \%(195)$ & $58.4 \%(149)$ & $70.8 \%(24)$ & 0.406 \\
\hline $\begin{array}{l}\text { Eye examination done by } \\
\text { ophthalmologist during } \\
\text { past } 24 \text { months }\end{array}$ & $86.2 \%(370)$ & $85.6 \%(195)$ & $86.8 \%(151)$ & $87.5 \%(24)$ & 0.726 \\
\hline $\begin{array}{l}\text { Annual influenza } \\
\text { vaccination done }\end{array}$ & $63.2 \%(375)$ & $62.4 \%(197)$ & $66.5 \%(152)$ & $50.0 \%(26)$ & 0.740 \\
\hline $\begin{array}{l}\text { Physical activity } \\
\text { recommendations given }\end{array}$ & $66.4 \%(378)$ & $59.3 \%(199)$ & $74.5 \%(153)$ & $73.1 \%(26)$ & 0.005 \\
\hline $\begin{array}{l}\text { Diet recommendations } \\
\text { given }{ }^{b}\end{array}$ & $46.1 \%(373)$ & $43.9 \%(198)$ & $50.3 \%(149)$ & $38.5 \%(26)$ & 0.700 \\
\hline \multicolumn{6}{|l|}{$\begin{array}{l}\text { Outcome-of-care } \\
\text { indicators }\end{array}$} \\
\hline $\begin{array}{l}\text { Knowledge of what HbA1C } \\
\text { is }\end{array}$ & $85.4 \%(349)$ & $86.7 \%(188)$ & $83.0 \%(141)$ & $90.0 \%(20)$ & 0.698 \\
\hline HbA1C value ${ }^{a}$ & $7.2 \pm 1.2(215)$ & $7.2 \pm 1.1(124)$ & $7.1 \pm 1.2(79)$ & $7.4 \pm 1.3(12)$ & 0.929 \\
\hline SF-12 MCS & $46.9 \pm 10.8(369)$ & $47.9 \pm 11.1(196)$ & $46.9 \pm 9.7(147)$ & $38.8 \pm 11.1(26)$ & 0.001 \\
\hline SF-12 PCS & $44.0 \pm 10.0(369)$ & $44.9 \pm 10.5(196)$ & $43.4 \pm 9.3(147)$ & $40.1 \pm 8.4(26)$ & 0.004 \\
\hline ADDQoL global score & $-1.4 \pm 1.5(380)$ & $-1.3 \pm 1.4(199)$ & $-1.4 \pm 1.4(155)$ & $-2.3 \pm 2.4(26)$ & 0.010 \\
\hline PACIC global score & $2.6 \pm 0.9(366)$ & $2.6 \pm 0.9(192)$ & $2.6 \pm 0.9(149)$ & $2.4 \pm 1.1(25)$ & 0.755 \\
\hline Self-efficacy score & $7.6 \pm 1.7$ (369) & $8.0 \pm 1.7(195)$ & $7.4 \pm 1.6(148)$ & $6.2 \pm 2.0(26)$ & 0.000 \\
\hline
\end{tabular}

Data are presented as percentage or mean \pm SD. Because of rounding, numbers may not add up to $100 \%$. Higher values are better for all outcomes of care except for $\mathrm{HbA1C}$ value and ADDQoL global score. P-values for trend were calculated with a non-parametric test for trend (nptrend command in Stata).

HbA1C: glycated haemoglobin A1C; SF-12-MCS: 12-item Short Form Health Survey - Mental Component Score; SF12-PCS: 12-item Short Form Health Survey - Physical Component Score; ADDQoL: Audit of Diabetes-Dependent Quality of Life 19; PACIC: Patient Assessment of Chronic Illness Care.

a: Among those knowing what $\mathrm{HbA} 1 \mathrm{C}$ is.

b: No time frame. 
Table 3: Association between functional health literacy (FHL) and process-of-care indicators: crude and adjusted odds ratios $(95 \% \mathrm{Cl}$; reference $=$ good level of $\mathrm{FHL})$

\begin{tabular}{|c|c|c|c|c|c|c|c|c|}
\hline & \multicolumn{4}{|c|}{ Unadjusted } & \multicolumn{4}{|c|}{ Adjusted } \\
\hline & \multicolumn{2}{|c|}{ Medium } & \multicolumn{2}{|c|}{ Poor } & \multicolumn{2}{|c|}{ Medium } & \multicolumn{2}{|c|}{ Poor } \\
\hline & OR & $(95 \% \mathrm{Cl})$ & OR & $(95 \% \mathrm{Cl})$ & OR & $(95 \% \mathrm{Cl})$ & OR & $(95 \% \mathrm{Cl})$ \\
\hline Annual lipid profile & 1.0 & $(0.4,2.6)$ & $\star \star \star *$ & *** & 1.1 & $(0.4,2.6)$ & *** & *** \\
\hline Annual urine test & 1.1 & $(0.6,1.9)$ & 0.8 & $(0.3,2.1)$ & 1.1 & $(0.6,2.0)$ & 0.7 & $(0.2,1.9)$ \\
\hline $\begin{array}{l}\text { Annual foot } \\
\text { examination }\end{array}$ & 1.0 & $(0.7,1.6)$ & 1.8 & $(0.7,4.4)$ & 1.0 & $(0.6,1.7)$ & 1.6 & $(0.6,4.2)$ \\
\hline $\begin{array}{l}\text { Eye examination by } \\
\text { ophthalmologist } \\
\text { during past } 24 \\
\text { months }\end{array}$ & 1.1 & $(0.6,2.0)$ & 1.2 & $(0.3,4.2)$ & 1.4 & $(0.7,2.8)$ & 0.9 & $(0.2,3.6)$ \\
\hline $\begin{array}{l}\text { Annual influenza } \\
\text { vaccination }\end{array}$ & 1.2 & $(0.8,1.9)$ & 0.6 & $(0.3,1.4)$ & 1.2 & $(0.7,2.0)$ & 0.6 & $(0.2,1.5)$ \\
\hline $\begin{array}{l}\text { Physical activity } \\
\text { recommendations }\end{array}$ & 2.0 & $(1.3,3.2)$ & 1.9 & $(0.7,4.6)$ & 1.9 & $(1.1,3.1)$ & 2.3 & $(0.8,6.2)$ \\
\hline $\begin{array}{l}\text { Diet } \\
\text { recommendations a }\end{array}$ & 1.3 & $(0.8,2.0)$ & 0.8 & $(0.3,1.8)$ & 1.5 & $(0.9,2.3)$ & 0.8 & $(0.3,1.9)$ \\
\hline
\end{tabular}


Table 4: Association between functional health literacy $(F H L)$ and outcome-of-care indicators: crude and adjusted $\beta$-coefficients $(95 \% \mathrm{Cl}$; reference $=$ good level of $\mathrm{FHL})$

\begin{tabular}{|c|c|c|c|c|c|c|c|c|}
\hline & \multicolumn{4}{|c|}{ Unadjusted } & \multicolumn{4}{|c|}{ Adjusted } \\
\hline & \multicolumn{2}{|c|}{ Medium } & \multicolumn{2}{|l|}{ Poor } & \multicolumn{2}{|c|}{ Medium } & \multicolumn{2}{|l|}{ Poor } \\
\hline & $\beta$-coef & $(95 \% \mathrm{Cl})$ & $\beta$-coef & $(95 \% \mathrm{Cl})$ & $\beta$-coef & $(95 \% \mathrm{Cl})$ & $\beta$-coef & $(95 \% \mathrm{Cl})$ \\
\hline $\begin{array}{l}\text { HbA1C } \\
\text { knowledge (349) }\end{array}$ & $0.7^{\mathrm{a}}$ & $(0.4,1.4)$ & $1.4^{\mathrm{a}}$ & $(0.3,6.3)$ & $0.7^{\mathrm{a}}$ & $(0.4,1.4)$ & $1.4^{\mathrm{a}}$ & $(0.3,7.0)$ \\
\hline $\begin{array}{l}\text { HbA1C value }{ }^{b} \\
(215)\end{array}$ & -0.1 & $(-0.4,0.3)$ & 0.3 & $(-0.4,1.0)$ & 0.007 & $(-0.3,0.3)$ & 0.3 & $(-0.4,1.0)$ \\
\hline SF-12 MCS (368) & -1.0 & $(-3.3,1.2)$ & -9.1 & $\begin{array}{l}(-13.5,- \\
4.8)\end{array}$ & -1.1 & $(-3.3,1.0)$ & -8.4 & $\begin{array}{l}(-12.5,- \\
4.2)\end{array}$ \\
\hline SF-12 PCS (369) & -1.6 & $(-3.7,0.5)$ & -4.9 & $(-8.9,-0.8)$ & -0.9 & $(-2.8,1.0)$ & -3.6 & $(-7.2,0.1)$ \\
\hline $\begin{array}{l}\text { ADDQoL global } \\
\text { score }(\mathbf{3 8 0})\end{array}$ & -0.2 & $(-0.5,0.1)$ & -1.1 & $(-1.7,-0.5)$ & -0.2 & $(-0.5,0.1)$ & 0.0 & $(-0.6,0.6)$ \\
\hline $\begin{array}{l}\text { PACIC global } \\
\text { score }(366)\end{array}$ & 0.1 & $(-0.1,0.3)$ & -0.1 & $(-0.5,0.2)$ & 0.1 & $(-0.1,0.3)$ & -0.4 & $(-0.8,0.0)$ \\
\hline $\begin{array}{l}\text { Self-efficacy } \\
\text { score (369) }\end{array}$ & -0.6 & $(-0.9,-0.2)$ & -1.7 & $(-2.4,-1.0)$ & -0.6 & $(-0.9,-0.2)$ & -1.8 & $(-2.5,-1.2)$ \\
\hline $\begin{array}{l}\text { Linear regression } \\
\text { age, gender, educ } \\
\text { HbA1C: glycated } \\
\text { 12-PCS: 12-item S } \\
\text { Quality of Life } 19 ; \\
\text { a: Logistic regress } \\
\text { b: Among those kn }\end{array}$ & $\begin{array}{l}\text { ith } \beta \text {-coe } \\
\text { tion, diab } \\
\text { aemoglok }\end{array}$ & $\begin{array}{l}\text { icient ( } \beta \text {-coef } \\
\text { tes treatmen } \\
\text { n A1C; SF-12 }\end{array}$ & $\begin{array}{l}\text { ent of Ch } \\
5 \% \mathrm{Cl} \text {. }\end{array}$ & $\begin{array}{l}\text { confidence in } \\
\text { abetes and n } \\
\text { tem Short Fo } \\
\text { Component } \\
\text { nic lllness C }\end{array}$ & $\begin{array}{l}m \text { Healt } \\
\text { score; A } \\
\text { re. }\end{array}$ & $\begin{array}{l}\text { unless otherv } \\
\text { morbidities. } \\
\text { Survey - Men } \\
\text { QoL: Audit }\end{array}$ & $\begin{array}{l}\text { se stated } \\
\text { Diabetes }\end{array}$ & $\begin{array}{l}\text { Adjusted for } \\
\text { ent Score; SF } \\
\text { Dependent }\end{array}$ \\
\hline
\end{tabular}

